\title{
Efeito das interações entre os íons amônio e nitrato na fisiologia do crescimento do amendoinzeiro
}

\author{
Marcos de Oliveira Ribeiro ${ }^{1}$, Cácio Luiz Boechat ${ }^{2}$, Maria das Graças da Silva Conceição ${ }^{1}$, Flavia Melo Moreira ${ }^{3}$, \\ Lucas de Oliveira Ribeiro ${ }^{1}$, Anacleto Ranulfo dos Santos ${ }^{4}$
}

\begin{abstract}
RESUMO
O amendoim é uma oleaginosa de grande importância para a região nordeste, pois geralmente é cultivado por pequenos produtores que, em média, não ultrapassam dez hectares e que visam a aumentar sua renda e a diversificar a produção. Este trabalho objetivou avaliar o efeito das relações entre formas nitrogenadas (amoniacal e nítrica) de adubação no desenvolvimento inicial do amendoinzeiro. Para isso foi utilizada solução nutritiva completa com concentração única de $210 \mathrm{mg} \mathrm{N} \mathrm{L}^{-1}$ fornecida em cinco proporções entre $\mathrm{NH}_{4}^{+}: \mathrm{NO}_{3}^{-}: 100: 0 ; 75: 25 ; 50: 50 ; 25: 75$ e 0:100. As unidades experimentais foram dispostas em delineamento inteiramente casualizado, com quatro repetições. As variáveis avaliadas foram: comprimento da parte aérea, comprimento da raiz, número de folhas, diâmetro do caule, massas de matérias fresca e seca da parte aérea e massas de matérias fresca e seca da raiz. Nas relações 100:0; 75:25 e 50:50, o amônio causou toxidez, reduzindo a fitomassa das plantas. Contudo, o nitrato (relação 0:100) causou menor redução, comparada com as reduções nas maiores concentrações do amônio. Nas relações 25:75 e 0:100, as plantas desenvolveram-se normalmente, apresentando maior fitomassa. $\mathrm{O}$ fornecimento exclusivo de nitrogênio na forma amoniacal não é a melhor opção para a nutrição do amendoinzeiro em fase inicial de crescimento.
\end{abstract}

Palavras-chave: Arachis hypogaea L., $\mathrm{NH}_{4}^{+}, \mathrm{NO}_{3}^{-}$, nitrogênio.

\section{ABSTRACT \\ Effect of interactions between ammonium and nitrate on the growth physiology of groundnut}

Peanut is a crop of great importance for the Northeast region, as it is usually grown by small farmers, who on average, do not exceed 10 ha of cultivated area and aim to increase its profitability and diversify the production. This study aimed to evaluate the effect of the relationship between nitrogen forms (ammonium and nitrate) in the initial growth of peanuts. Complete nutrient solution was used with a single concentration of $210 \mathrm{mg} \mathrm{N} \mathrm{L}^{-1}$ provided in five proportions of $\mathrm{NH}_{4}^{+}: \mathrm{NO}_{3}^{-}: 100: 0,75: 25,50: 50,25: 75$ and $0: 100$. The experimental units were arranged in completely randomized design, with four replications. The variables evaluated were: shoot length, root length, leaf number, stem diameter, fresh and dry shoot and fresh weight and dry root. The 100:0, 75:25 and 50:50 ratio resulted in decreasing peanut phytomass by ammonium toxicity. However, the 0:100 ratio, nitrate caused a smaller decreased compared to reductions in the highest ammonium concentrations. In the 25:75 and 0:100 ratio, plants grow normally with higher phytomass. The supply of nitrogen in the $\mathrm{NH}_{4}{ }^{+}$form only is not a good option for the nutrition of peanuts in early growth stages.

Key words: Arachis hypogaea $\mathrm{L} ., \mathrm{NH}_{4}^{+}, \mathrm{NO}_{3}^{-}$, nitrogen.

Recebido para publicação em 01/06/2012 e aprovado em 12/09/2012.

${ }^{1}$ Graduandos em Agronomia. Centro de Ciências Agrárias, Ambientais e Biológicas, Universidade Federal do Recôncavo da Bahia, Rua Rui Barbosa, 710, Centro, 44380-000, Cruz das Almas, Bahia, Brasil. marcos_oliveira05@hotmail.com; liagal_73@hotmail.com; lucasdeoliveira2@yahoo.com.br

${ }^{2}$ Engenheiro-Agrônomo, Mestre. Doutorando do Departamento de Ciência do Solo, Universidade Federal do Rio Grande do Sul, Avenida Bento Gonçalves, 7712 , Caixa Postal 15100, 91540-000, Porto Alegre, Rio Grande do Sul, Brasil. clboechat@hotmail.com (autor para correspondência).

${ }^{3}$ Graduanda em Engenharia florestal. Centro de Ciências Agrárias, Ambientais e Biológicas, Universidade Federal do Recôncavo da Bahia, Rua Rui Barbosa, 710 , Centro, 44380000, Cruz das Almas, Bahia, Brasil. fmmoreira_ef@yahoo.com.br

${ }^{4}$ Engenheiro-Agrônomo, Doutor. Centro de Ciências Agrárias, Ambientais e Biológicas, Universidade Federal do Recôncavo da Bahia, Rua Rui Barbosa, 710, Centro, 44380-000, Cruz das Almas, Bahia, Brasil. anacleto@ufrb.edu.br 


\section{INTRODUÇÃO}

O amendoim (Arachis hypogaea L.), por sua palatabilidade e alto valor nutritivo, além de elevado teor de óleo, é bastante utilizado e consumido no Brasil e em vários países do mundo. Seu cultivo estende-se de norte a sul do País, graças a sua ampla adaptabilidade em diferentes condições edafoclimáticas (Peixoto et al., 2008).

Na região Nordeste, o amendoinzeiro geralmente é cultivado por pequenos produtores que, em média, não ultrapassam 10 ha (Santos et al., 2006) e que visam a aumentar sua renda e a diversificar a produção (Oliveira $e t$ al., 2010).

O amendoinzeiro é uma planta incomum, visto que suas vagens desenvolvem-se no solo, ou seja, sua frutificação é hipógea, motivo pelo qual o cuidado com o solo para o bom rendimento da cultura desta planta é ainda maior, pois os frutos absorvem nutrientes diretamente do substrato (solo) e exigem uma porosidade elevada, para o processo de trocas respiratórias do solo com a atmosfera, bem como para a boa qualidade da vagem produzida (Freire et al., 2007).

A nutrição e a adubação apresentam aspectos controvertidos, na cultura do amendoinzeiro, porém a falta de qualquer elemento essencial à planta afeta seu crescimento, desenvolvimento e produção (Freire et al., 2007).

O nitrogênio é o nutriente considerado mais crítico para a produtividade agrícola, sendo o solo importante reservatório deste elemento, por se tratar do principal elo entre os componentes da biosfera (Borges et al., 2007). Nas plantas, o nitrogênio representa de 1 a $6 \%$ da sua matéria seca, e pode ser absorvido sob diferentes formas (Havlin et al., 2005). Porém, as formas preferenciais de $\mathrm{N}$ absorvido e metabolizado pelas plantas são aquelas mais abundantes na solução do solo: nitrato e amônio (Taiz \& Zeiger, 2009).

Em algumas culturas, existe efeito negativo do íon amônio sobre o crescimento, o que se atribui à necessidade de utilização dos carboidratos produzidos, prioritariamente, para a rápida assimilação do amônio absorvido, com vistas a evitar sua acumulação e consequentes problemas de toxicidade, relacionados com alterações no $\mathrm{pH}$ celular e desbalanço iônico e hormonal (Britto \& Kronzucker, 2002).

A absorção radicular de amônio depende de sua única entrada, mediada por um transportador, quando levado ao citoplasma da célula, ocasiona um desequilíbrio eletrostático entre os meios intra e extracelular, o que faz que a célula promova um fluxo contrário de cargas positivas, a fim de alcançar a neutralidade (Silva et al., 2010).

Este trabalho teve por objetivo avaliar o efeito das interações de diferentes proporções entre as formas nitrogenadas, a nítrica e a amoniacal, no crescimento do amendoinzeiro.

\section{MATERIAL E MÉTODOS}

O experimento foi conduzido, no período de dezembro de 2011 a janeiro de 2012, em casa de vegetação do Centro de Ciências Agrárias, Ambientais e Biológicas, da Universidade Federal do Recôncavo da Bahia (Cruz das Almas, BA), cujas coordenadas geográficas são: $12^{\circ}$ $40^{\prime} 12^{\prime \prime} \mathrm{S}, 39^{\circ} 06^{\prime} 07^{\prime \prime} \mathrm{W}$, com altitude de $220 \mathrm{~m}$, temperatura média anual de $24,5^{\circ} \mathrm{C}$ e clima tropical subúmido a seco (SEI, 2012).

As unidades experimentais (UEs) foram constituídas de recipientes de polietileno com capacidade para $1 \mathrm{~L}$. Como substrato, foi utilizada areia lavada, objetivando criar um meio adequado para o desenvolvimento e a distribuição do sistema radicular do amendoinzeiro.

O experimento foi conduzido em solução nutritiva, composta por macro e micronutrientes, com $\mathrm{pH}$ ajustado a 5,6, utilizando-se $\mathrm{HCl} 0,01 \mathrm{M}$ ou $\mathrm{NaOH} 0,01 \mathrm{M}$. Os tratamentos seguiram a concentração única de $210 \mathrm{mg} \mathrm{N} \mathrm{L}^{-1}$ sugerida por Hoagland \& Arnon (1950), fornecida em cinco proporções entre nitrogênio amoniacal e nítrico $\left(\mathrm{NH}_{4}^{+}: \mathrm{NO}_{3}{ }^{-}\right): 100: 0 ; 75: 25 ; 50: 50 ; 25: 75 ; 0: 100$. As UEs foram dispostas em delineamento inteiramente casualizado, com quatro repetições, sendo cada UE constituída por duas plantas.

As sementes foram embebidas em água destilada, durante 24 horas, sendo semeadas em meio estéril, contendo somente água destilada. Aos seis dias após a emergência (DAE), quando atingiram $7 \mathrm{~cm}$ de altura, foram transplantadas duas mudas por UE e iniciado o fornecimento da solução nutritiva. O recipiente foi previamente preparado com 1,5 $\mathrm{L}$ de areia previamente purificada, a que se adicionaram $200 \mathrm{ml}$ de solução nutritiva com $100 \%$ da força iônica. Durante a condução do experimento, as soluções foram diariamente verificadas, sob o critério de manter o $\mathrm{pH}$ entre 5,5 e 6,0. Sempre que se verificou, por meio de pesagem da UE, redução de $\pm 150 \mathrm{ml}$ no nível da solução, causada pela evapotranspiração, esta foi reposta com água destilada.

Depois de colhidas, aos $37 \mathrm{DAE}$, as plantas foram separadas em parte aérea e raiz. As raízes foram lavadas em água destilada, para retirada do substrato aderido, para, então, serem secas em papel absorvente. As plantas foram avaliadas quanto ao comprimento da parte aérea, distância do colo ao ápice do meristema apical (CPA); comprimento da raiz (CR), distância desde o ápice da raiz principal até a base da planta; número de folhas (NF); diâmetro do caule (DC), medido com paquímetro digital graduado; massa de matéria fresca da parte aérea (MFPA); massa de matéria fresca da raiz (MFR).

Para avaliação das variáveis relacionadas com a massa de matéria seca, as plantas foram acondicionadas indi- 
vidualmente em sacos de papel, colocadas em estufa, com circulação forçada de ar, a $65^{\circ} \mathrm{C}$, durante 72 horas, após o que foram analisadas as variáveis massa de matéria seca da parte aérea (MSPA) e massa de matéria seca da raiz (MSR).

Os dados foram submetidos à análise de variância (ANOVA). O teste de Tukey ( $\mathrm{p}<0,05$ ) foi utilizado para separar os efeitos das médias. As análises foram feitas, empregando-se o programa estatístico SISVAR ${ }^{\circledR} 5.3$ (Ferreira, 2008).

\section{RESULTADOS E DISCUSSÃO}

Não ocorreu efeito significativo dos tratamentos para comprimento da parte aérea (CPA) e para diâmetro do caule (DC), no entanto, foram observadas respostas significativas para o comprimento de raiz (CR), massas de matérias fresca e seca da parte aérea e da raiz (MFPA, MSPA, MFR e MSR) e número de folhas (NF) ( $\mathrm{p}<0,05)$, demonstrando-se, assim, que a cultura do amendoinzeiro responde às diferentes relações entre os íons amônio e nitrato $\left(\mathrm{NH}_{4}^{+}: \mathrm{NO}_{3}^{-}\right)$na solução nutritiva (Figura 1$)$.

Observando as Figuras $1 \mathrm{~A}$ a $1 \mathrm{H}$, verifica-se que as relações $\mathrm{NH}_{4}^{+}: \mathrm{NO}_{3}{ }^{-}$de 25:75 e 0:100 obtiveram os melhores resultados no conjunto das variáveis fisiológicas selecionadas. O comprimento da parte aérea (CPA) e o diâmetro do caule (DC) não apresentaram diferenças significativas $(\mathrm{p}<0,05)$ entre as proporções de amônio e nitrato, com valores variando entre 9,50 e 13,65 cm, para CPA, e entre 0,28 e 0,40 cm, para DC (Figuras 1A e 1C). Resultados semelhantes foram encontrados por Oliveira et al. (2008), estudando o crescimento inicial do milho de pipoca. Estes autores não observaram diferenças significativas na altura das plantas com as diferentes relações $\mathrm{NH}_{4}^{+}: \mathrm{NO}_{3}^{-}$.

Diferentes hipóteses foram elaboradas para explicar as causas da toxidez por $\mathrm{NH}_{4}^{+}$, entre as quais a relação da entrada excessiva de $\mathrm{NH}_{4}{ }^{+}$com a despolarização da membrana plasmática e do tonoplasto, a acidificação das organelas celulares, na tentativa de manter o potencial elétrico das membranas, mudanças no status de carboidratos das plantas, desacoplamento da fotofosforilação e, consequentemente, distúrbios fisiológicos que levam à morte das células e do tecido (Britto \& Kronzucker, 2002, 2005).

A diminuição do comprimento da raiz (Figura 1B) na maior concentração de $\mathrm{NH}_{4}{ }^{+}$contraria a hipótese de que as raízes diminuem o seu crescimento quando existe água e nutrientes, principalmente $\mathrm{N}$, disponíveis no solo (Andriolo et al., 2006). Todavia, reiterando o que foi afirmado por Thornley (1998), o crescimento das raízes tem sido considerado como dependente do balanço de carbono/nitrogênio entre a parte aérea e os compartimentos de raiz.
O uso de amônio em maiores proporções (100:0; 75:25 e 50:50) apresentou os piores resultados para as variáveis comprimento de raiz (CR), número de folhas (NF), e todas as relacionadas com fitomassas fresca e seca da planta de amendoim (Figuras 1B, 1D, 1E a 1H). Esse efeito tem sido atribuído ao fato de que os carboidratos translocados das folhas para as raízes de absorção são utilizados, prioritariamente, como esqueletos de carbono e energia para o processo de assimilação do íon amônio, a fim de evitar seu acúmulo em níveis tóxicos, e não para os processos associados ao crescimento desse órgão (Lewis et al., 1989). Holzschuh et al. (2011) observaram, por meio da análise da seiva do xilema da planta de arroz, que o amônio afeta negativamente a absorção dos íons potássio, cálcio e magnésio, em magnitude dependente da sua concentração no meio.

Para o NF, houve diferenças significativas entre os tratamentos, com melhor resultado na proporção 25:75, alcançando valor médio de 6,75 folhas planta por planta (Figura 1D). Resultados semelhantes foram encontrados por Andriolo et al. (2006), com a redução do número de folhas em função do aumento da concentração de $\mathrm{NH}_{4}^{+}$na solução. Estudando a atividade fotossintética da cultura da mandioca, Cruz et al. (2008) associaram fatores ligados à menor atividade, em virtude da ação negativa do íon $\mathrm{NH}_{4}{ }^{+}$sobre a condutância estomática na planta.

Nas Figuras 1E e 1F observam-se reduções de 81,7 e 71,05\% para MFPA e MSPA, na relação 100:0, e de 81,13 e 78,95\%, na relação 75:25, em comparação com a redução observada na relação 25:75. Andriolo et al. (2006) observaram que o peso fresco da alface foi reduzido pela salinidade da solução nutritiva, em um intervalo de concentração entre cerca de 1,0 e 5,0 dS m ${ }^{-1}$, em razão da maior concentração do $\mathrm{NH}_{4}^{+}$corroborando os resultados encontrados por Godoi et al. (2007), estudando concentrações de amônio, associadas à nutrição de dois cultivares de almeirão. Esta redução foi relacionada com restrições no fluxo de água da planta, por efeito da salinidade (Andriolo et al., 2006) e com a redução na atividade da enzima nitrato redutase (Silva et al., 2011), causada pelo íon amônio. Isto implica restrições tanto da síntese de massa de matéria seca como da absorção de água pelas raízes. Na literatura, os efeitos de toxicidade $\mathrm{NH}_{4}^{+}$têm sido atribuídos à redução ou inibição da absorção de cátions, especialmente o potássio, como uma consequência do desequilíbrio de íons (Kotsiras et al., 2002).

Semelhantemente, houve redução de 26,23 e 21,05\%, na relação 0:100, com apenas nitrato na composição, e de 50,94 e 56,58\%, na relação 50:50, para MFPAe MSPA, em comparação com a redução observada na relação 25:75 (Figuras 1E e 1F). 

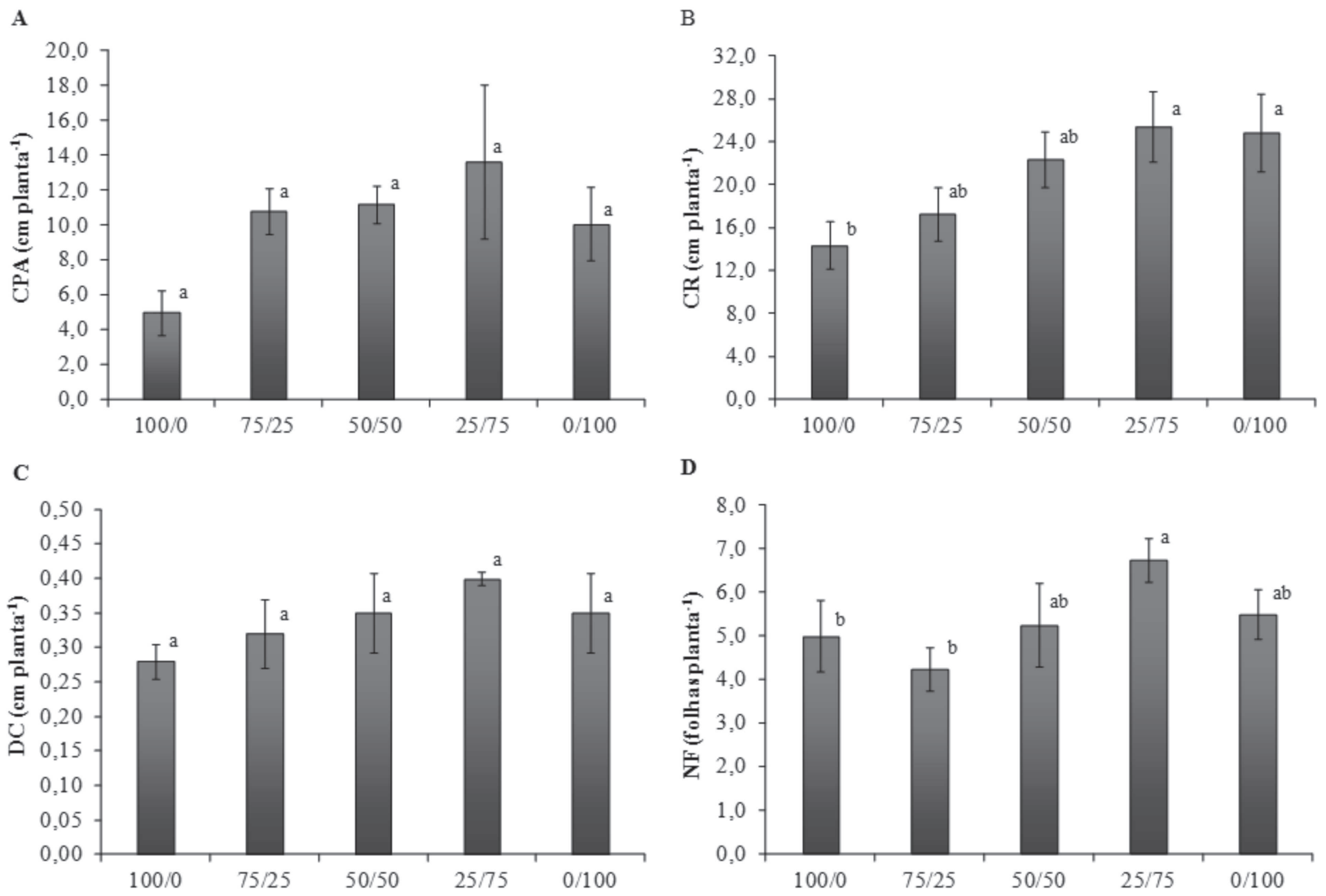

D

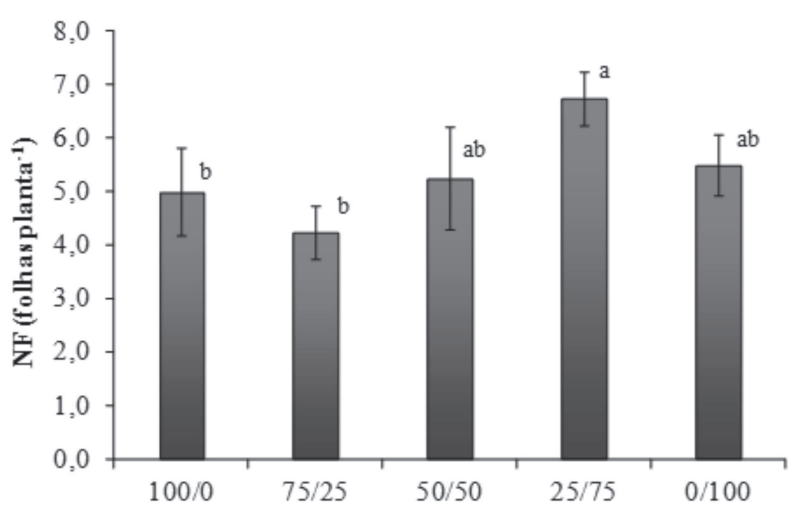

$\mathbf{E}$

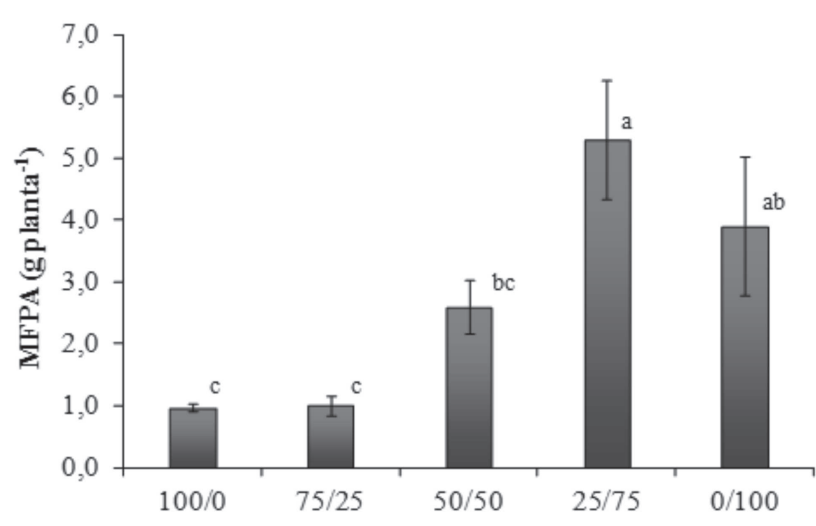

$\mathbf{F}$

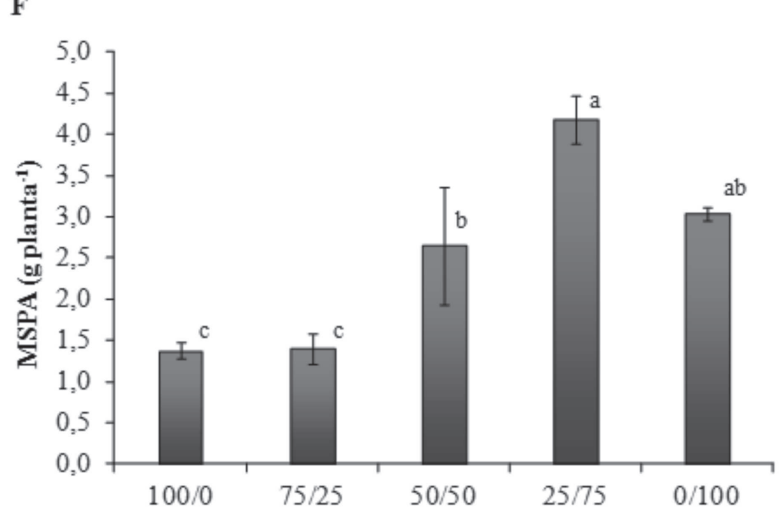

G

H
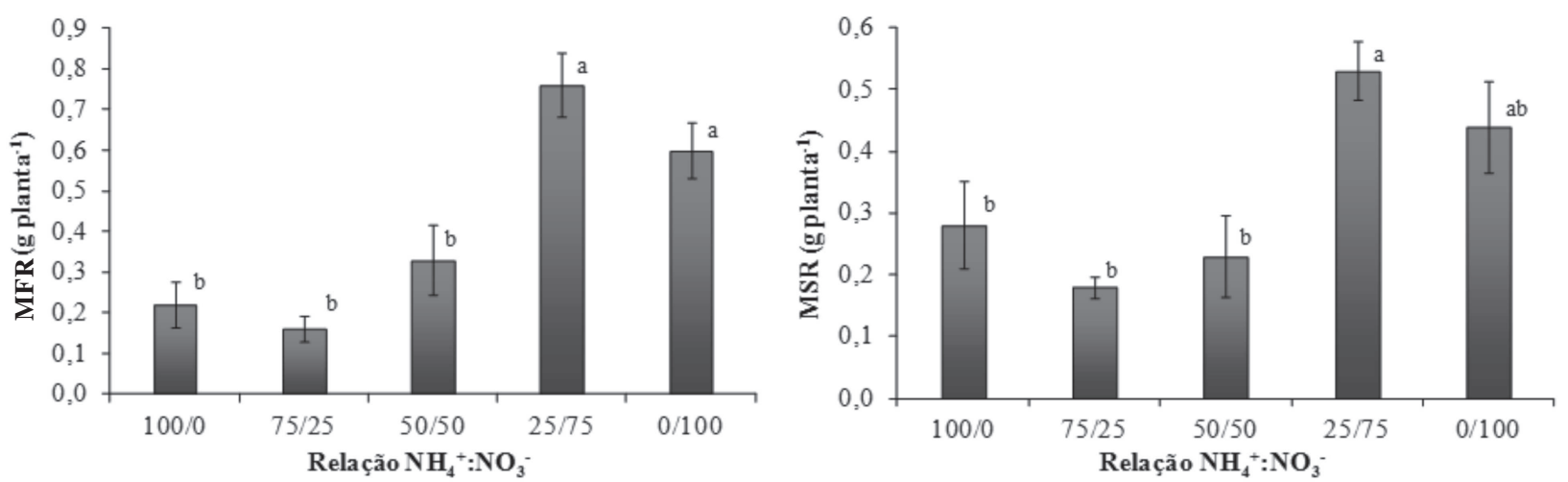

Figura 1. Valores médios para comprimento da parte aérea (CPA, A), comprimento da raiz (CR, B), diâmetro do caule (DC, C), número de folhas (NF, D), massas de matérias fresca e seca da parte aérea (MFPA, E e MSPA, F) e massas de matérias fresca e seca da raiz (MFR, G e MSR, H) do amendoim fertilizado com diferentes relações $\mathrm{NH}_{4}^{+}: \mathrm{NO}_{3}^{-}$. Médias seguidas de mesma letra não diferem entre si pelo teste de Tukey $(\mathrm{p}<0,05)$. 
De acordo com Andrade et al. (2001), a atividade da enzima redutase do nitrato é aumentada significativamente quando lhe são oferecidas ambas as fontes de $\mathrm{N}\left(\mathrm{NH}_{4}^{+}\right.$ e $\mathrm{NO}_{3}^{-}$), indicando certa ação estimulatória do íon amônio na atividade da enzima, o que explica o fato de os melhores resultados para as variáveis fisiológicas do amendoim não terem sido obtidos na relação 0:100, que corresponde ao $\mathrm{N}$ fornecido $100 \%$ na forma nítrica. Contudo, o aumento da percentagem de $\mathrm{NH}_{4}^{+}$é prejudicial, diminuindo o teor de nitrato no pecíolo de plantas de algodão e a atividade da enzima redutase do nitrato, e aumentando o conteúdo de nitrogênio amoniacal em todos os órgãos do algodão (Dong et al., 2004).

Kronzucker et al. (2000) e Britto et al. (2001) propuseram outro mecanismo, que estaria associado à toxidez por $\mathrm{NH}_{4}^{+}$. Para esses autores, o fato de a absorção excessiva de $\mathrm{NH}_{4}{ }^{+}$ocorrer por transportadores de baixa afinidade e de ocupar canais de outros cátions indica que esse possa ser o motivo de se criar um mecanismo de efluxo do $\mathrm{NH}_{4}^{+}$ para fora da célula. Esse mecanismo foi chamado, por esses autores, de Ciclagem Fútil, e o efeito resultante é um elevado gasto energético, necessário para bombear o excesso de $\mathrm{NH}_{4}^{+}$para fora da célula.

Conforme Kronzucker et al. (2000) e Britto et al. (2001), cerca de $80 \%$ do amônio absorvido pode sofrer efluxo por Ciclagem Fútil e o elevado consumo de energia na forma de ATP resulta no aumento da respiração nas raízes, causando redução no seu crescimento. Este mecanismo, provavelmente, foi responsável pela diminuição do comprimento de raízes (Figuras 1B, $1 \mathrm{G} \mathrm{e} 1 \mathrm{H}$ ), de aproximadamente 43,$5 ; 32,2 ; 12,2$ e $2,2 \%$, na relação $100: 0 ; 75: 25 ; 50: 50$ e $0: 100\left(\mathrm{NH}_{4}^{+}: \mathrm{NO}_{3}^{-}\right)$, respectivamente, quando comparadas com os efeitos observados na relação 25:75. Consequentemente, este efeito refletiu-se diretamente nos valores das variáveis MFR e MSR, relacionadas com a fitomassa de raiz do amendoinzeiro.

Trabalhando com plantas de arroz em solução, Holzschuh et al. (2011) observaram comportamento semelhante para os efeitos da relação 25:75, com elevado incremento do comprimento e da fitomassa de raízes, em comparação com os das demais relações. Contudo, observou-se que o aumento de nitrato, até a relação 25:75, foi benéfico no alongamento de raiz, não se refletindo da mesma forma na fitomassa de raiz, com exceção da relação 0:100 (Figuras 1G e 1H). Entretanto, essa ligeira redução mantém relação com o gasto de ATP necessário para a assimilação, síntese e translocação de fotoassimilados da parte aérea para as raízes (Reddy \& Menary, 1990).

Portanto, a variação do crescimento das raízes entre os tratamentos pode ser atribuída ao efeito do íon $\mathrm{NO}_{3}$ sobre o estímulo ao desenvolvimento de raízes e, também, sobre a redução da toxidez por $\mathrm{NH}_{4}^{+}$(Holzschuh et al., 2011).

\section{CONCLUSÃO}

O fornecimento de $\mathrm{N}$ nas formas nítrica e amoniacal é imprescindível para o melhor crescimento de amendoinzeiro, na fase inicial, sendo que a presença de amônio acima da relação 25:75 $\left(\mathrm{NH}_{4}^{+}: \mathrm{NO}_{3}^{-}\right)$prejudica o crescimento inicial da planta, com relação às variáveis fisiológicas avaliadas; entretanto, a combinação de $\mathrm{NH}_{4}{ }^{+}$e $\mathrm{NO}_{3}{ }^{-}$nas relações 25:75 e 0:100 proporcionou maior produção de fitomassa da planta do amendoim.

O fornecimento exclusivo de nitrogênio na forma amoniacal não é a melhor opção para a nutrição do amendoim em fase inicial de crescimento.

\section{REFERÊNCIAS}

Andrade SRM, Sant'Anna R, Cambraia J \& Mosquim PR (2001) Efeito da proporção de $\mathrm{NH}_{4}^{+} / \mathrm{NO}_{3}{ }^{-}$na distribuição de nitrato e na atividade in vitro da redutase do nitrato em plantas de Panicum maximum. Planaltina, Embrapa Cerrados. 16p. (Boletim de pesquisa e Desenvolvimento, 12).

Andriolo JL, Godoi RS, Cogo CM, Bortolotto OC, Luz GL \& Madaloz JC (2006) Growth and development of lettuce plants at high $\mathrm{NH}_{4}^{+}: \mathrm{NO}_{3}$ - ratios in the nutrient solution. Horticultura Brasileira, 24:353-355.

Borges WL, Silva CER, Xavier GR \& Rumjanek NG (2007) Nodulação e fixação biológica de nitrogênio de acessos de amendoim com estirpes nativas de rizóbios. Revista Brasileira de Ciências Agrárias, 2:32-37.

Britto DT, Siddiqi MY, Glass ADM \& Kronzucker HJ (2001) Futile transmembrane $\mathrm{NH}_{4}^{+}$cycling: A cellular hypothesis to explain ammonium toxicity in plants. Proceedings of the National Academy of Sciences of the United States of America, 98:42554258 .

Britto DT \& Kronzucker HJ (2002) $\mathrm{NH}_{4}{ }^{+}$toxicity in higher plants: a critical review. Journal of Plant Physiology, 159:567-584.

Britto DT \& Kronzucker HJ (2005) Plant nitrogen transport and its regulation in changing soil environments. Journal of Crop Improvement, 15:01-23.

Cruz JL, Pelacani CR \& Araújo WL (2008) Influência do nitrato e do amônio sobre a fotossíntese e a concentração de compostos nitrogenados em mandioca. Ciência Rural, 38:643-649.

Dong $\mathrm{H}$, Li J \& Li C (2004) Influence of different $\mathrm{NH}_{4}^{+} / \mathrm{NO}_{3}^{-}$ ratios on nitrogen metabolism of cotton. Ying yong sheng tai xue bao $=$ The Journal of Applied Ecology, 15:728-730.

Ferreira DF (2008) SISVAR: Um programa para análises estatísticas e ensino de estatística. Revista Científica Symposium, 6:3641 .

Freire MLF, Beltrão NEM, Rao TVR \& Menezes HEA (2007) Análise de crescimento não-destrutiva do amendoinzeiro submetido a doses de $\mathrm{CaSO}_{4}$ e $\mathrm{P}_{2} \mathrm{O}_{5}$. Revista Brasileira de Ciências Agrárias, 2:193-199.

Godoi RS, Andrioloi JL, Madalózi JCC, Janischi DI \& Barros CAP (2007) Crescimento e produtividade do almeirão em concentrações de $\mathrm{N}$ total contendo $\mathrm{NH}_{4}^{+}$na solução nutritiva. Ciência Rural, 37:1170-1173.

Havlin JL, Beaton JD, Tisdale SL \& Nelson WL (2005) Soil fertility and fertilizers: an introduction to nutrient management. $7^{\text {th }}$ ed. New Jersey, Prentice Hall. 515p. 
Hoagland DR \& Arnon DI (1950) The water-culture method for growing plants without soil. Berkeley, University of California. 32p. (Circular, 347).

Holzschuh MJ, Bohnen H, Anghinoni I, Pizzolato TM, Carmona FC \& Carlos FS (2011) Absorção de nutrientes e crescimento do arroz com suprimento combinado de amônio e nitrato. Revista Brasileira de Ciência do Solo, 35:1357-1366.

Kotsiras A, Olympios CM, Drosopoulos J \& Passam HC (2002) Effects of nitrogen form and concentration on the distribution of ions within cucumber fruits. Scientia Horticulturae, 95:175183

Kronzucker HJ, Glass ADM, Siddiqi MY \& Kirk GJD (2000) Comparative kinetic analysis of ammonium and nitrate acquisition by tropical lowland rice: Implications for rice cultivation and yield potential. New Phytologist, 145:471-476.

Lewis OAM, Leidi EO \& Lips SH (1989) Effect of nitrogen source on growth response to salinity stress in maize and wheat. New Phytologyst, 111:55-160.

Oliveira FA, Medeiros JF, Maracajá PB, Oliveira MKT \& Guimarães IP (2008) Desenvolvimento inicial do milho pipoca cultivado sob diferentes relações $\mathrm{NH}_{4}^{+}: \mathrm{NO}_{3}$. Revista Caatinga, 21:197-201

Oliveira TMM, Queiroga RCF, Nogueira FP, Moreira JN \& Santos MA (2010) Produção de cultivares decumbentes de amendoim submetidas a distintos espaçamentos. Revista Caatinga, 23:149154.
Peixoto CP, Goncalves JA, Peixoto MFSP \& Carmo DO (2008) Características agronômicas e produtividade de amendoim em diferentes espaçamentos e épocas de semeadura no recôncavo baiano. Bragantia, 67:563-568.

Reddy KS \& Menary RC (1990) Nitrate reductase and nitrate accumulation in relation to nitrate toxicity in Boronia megastigma. Physiologia Plantarum, 78:430-434.

Santos RC, Gondim TMS \& Freire RMM (2006) Sistema de produção. Disponível em: <http:// sistemasdeproducao.cnptia.embrapa.br $>$. Acessado em: 15 de janeiro de 2012.

SEI - Superintendência de Estudos Econômicos e Sociais da Bahia (2012) Sistema de dados estatísticos. Disponível em: <http:// w w w . s e i . b a . g o v . b r / s i d e / frame_tabela.wsp?tmp.volta $=$ sg $46 \&$ tmp.tabela $=t 106>$. Acessado em: 09 de fevereiro de 2012.

Silva PCC, Couto JL \& Santos AR (2010) Efeito dos íons amônio e nitrato no desenvolvimento do girassol em solução nutritiva. Revista da FZVA, 17:104-114.

Silva SM, Oliveira LJ, Faria FP, Reis EF, Carneiroi MAC \& Silva SM (2011) Atividade da enzima nitrato redutase em milho cultivado sob diferentes níveis de adubação nitrogenada e potássica. Ciência Rural, 41:1931-1937.

Taiz L \& Zeiger E (2009) Fisiologia vegetal. $4^{\mathrm{a}}$ ed. Porto Alegre, Artmed. 719p.

Thornley JHM (1998) Modelling shoot: root relations: the only way forward? Annals of Botany, 81:165-171. 